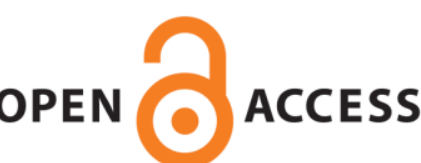

OPEN ACCESS

UWS Academic Portal

\title{
Metatarsal strapping tightness effect to vertical jump performance
}

Zhang, Yan; Baker, Julien S.; Ren, Xuejun; Feng, Neng; Gu, Yaodong

Published in:

Human Movement Science

DOI:

10.1016/j.humov.2015.03.013

Published: 01/06/2015

Document Version

Peer reviewed version

Link to publication on the UWS Academic Portal

Citation for published version (APA):

Zhang, Y., Baker, J. S., Ren, X., Feng, N., \& Gu, Y. (2015). Metatarsal strapping tightness effect to vertical jump performance. Human Movement Science, 41, 255-264. https://doi.org/10.1016/j.humov.2015.03.013

\section{General rights}

Copyright and moral rights for the publications made accessible in the UWS Academic Portal are retained by the authors and/or other copyright owners and it is a condition of accessing publications that users recognise and abide by the legal requirements associated with these rights.

\section{Take down policy}

If you believe that this document breaches copyright please contact pure@uws.ac.uk providing details, and we will remove access to the work immediately and investigate your claim. 


\title{
Metatarsal strapping tightness effect to vertical jump performance
}

\author{
Yan Zhang', Julien Baker', Xuejun Ren ${ }^{3}$, Neng Feng ${ }^{4}$, Yaodong $\mathbf{G u}^{1 *}$ \\ 1Faculty of Sports Science, Ningbo University, China \\ 2 School of Science and Sport, University of the West of Scotland, UK \\ 3School of Engineering, Liverpool John Moores University, UK \\ 4 Rehabilitation Center, Ningbo Ninth Hospital, China
}

\begin{abstract}
The study investigated the effect of metatarsal strapping on vertical jump performance and evaluated the difference in lower limb kinematics and electromyographic signal (EMG) between different strapping force levels. Twelve male callisthenic athletes completed single vertical jump from a squat posture with hands on hips under three conditions as non-strapping (NS), moderate strapping (MS) and high strapping (HS) round metatarsals. Ground reaction force (GRF) was recorded with KISTLER force platform to calculate the vertical jump height. Angles of ankle, knee and hip were measured with VICON motion analysis system and EMG data were recorded with mega6000 system. Data showed that jump height was significantly higher under HS than NS condition. Compared with NS, ankle inversion decreased significantly during take-off and external rotation increased significantly during landing with MS. Significant difference was also found in the muscle activity of tibialis anterior between non-strapping and strapping conditions.
\end{abstract}

Keywords: metatarsal strapping; lower limb kinematics; EMG; vertical jump performance

\section{Introduction}

Human body compression equipment has been widely used amongst athletes of all levels as a way to improve performance. For example, De Glanville et al. (2012) indicated that well-trained endurance athletes who wore full-leg-length compressive garment achieved a substantially higher average power output in the subsequent 40-kM cycling time trial. Kraemer et al. (1998) noted that compression shorts effectively reduced the muscle oscillation upon impact during landing and increased joint position sense; consequently, the mean power output during repetitive maximal jump was enhanced. Single countermovement jump height of experienced athletes wearing thicker compressive shorts with additional elastic force increased significantly by $2.4 \mathrm{~cm}$ than that of the control (Doan et al., 2003). Wearing 
below-knee compression stockings has shown to improve running performance of moderately trained athletes (Kemmler et al., 2009). Another work also reported that recreational athletes completed 5-km running race faster with elastic tights $(30 \mathrm{~mm}$ $\mathrm{Hg}$ ) at ankle by extending stride length (Chatard, 1998).

All these works reported on compressive interventions were applied to lower limb joints as hip, knee and ankle; no systematic work has been conducted similar approach on the metatarsophalangeal joint (MTP), probably due to the difficulty with its smaller anatomical structure and muscle group around such as flexor digitorum longus or extensor digitorum longus. MTP is known to play an important role in push-off/take-off as the terminal segment to resist external moment produced by GRF during locomotion (Goldmann \& Brüggemann, 2012). Previous studies reported that the peak MTP plantar flexion moments were about $60 \mathrm{Nm}$ and $110 \mathrm{Nm}$ during running and sprint, respectively, and this moment was greater than $100 \mathrm{Nm}$ during long jump (Stefanyshyn \& Nigg, 1998; Stefanyshyn \& Nigg, 1997).

Research works on MTP began to attract particular attention since mid-1990s, in early works; Stefanyshyn et al. (1997) investigated energy generation and absorption at this joint. The authors indicated that MTP absorbed energy whereas generated no or very little energy during take-off since the joint remained to be dorsiflexion almost throughout the stance phase, and could not transform to plantar-flexion until the toes off the ground (Stefanyshyn \& Nigg, 1997). The work showed that MTP absorbed, on average, 24J energy during one-legged vertical jump, and the jump height would increase approximately $3.5 \mathrm{~cm}$ with a body mass of $70 \mathrm{~kg}$ for such amount of energy (Stefanyshyn \& Nigg, 1998). Base on this case, reducing energy absorption at MTP may potentially be a viable approach to enhance performance. Research on intervention at metatarsals such as footwear modification has been reported to be helpful in reducing energy loss at this joint. One example is to increase midsole longitudinal bending stiffness. Stefanyshyn et al. (2000) showed that the vertical jump height could be increased significantly when wearing shoe with stiffer midsole on 
account of decreased energy absorption at MTP, whereas energy generation and absorption at ankle, knee, and hip joints were not influenced by midsole stiffness. In another work, Roy et al. (2006) reported that stiffened MTP improved running economy without the reduction of energy loss, which was associated with the principle of optimizing musculoskeletal system. Athletes are capable of manipulating equipment to optimize contractile properties of the muscles such as relationship of force-length and force-velocity leading to performance enhancement (Nigg et al., 2000). In addition, one work demonstrated that, in order to adapt gait with metatarsals intervention, ankle and knee angles would change notably (Wu et al., 2001). Similarly, Laroche et al. (2006) indicated that there was a negative relationship between maximal knee and hip flexion and underlying MTP dorsal flexion range of motion (ROM) during walking.

Evident from these published works, it is highly likely that sports performance can be improved through adding proper intervention on metatarsals with a proper mechanism. Meanwhile, the intervention may also induce compensatory motion of ankle, knee and hip. Different from using compression garments or shoes with stiffer midsole, this study used a much simpler elastic compression bandage to strap the metatarsals to explore the effect of metatarsal strapping on vertical jump height and lower limb kinematics. To link the muscle activity and the jump height, EMG of four main lower limb muscles were measured. It was hypothesised that metatarsal strapping would not greatly alter lower limb kinematics; but increased metatarsal strapping magnitude would increase jump height and decrease the total intensity of lower limb muscular activity, which leads to optimum effects.

\section{Method}

\subsection{Participants}

Twelve healthy male callisthenic athletes (age: $20.71 \pm 1.38$ years, height: $1.74 \pm 0.04 \mathrm{~m}$, weight: $66.26 \pm 2.84 \mathrm{~kg}$ ) were selected in this study. All the participants have excellent ability of posture control and coordination skills with the average training experience 
of 2.14 \pm 1.35 years. All the athletes are free from any injuries that could affect the motion during vertical jump. All subjects provided informed consent for inclusion in the study, which was approved by the Human Ethics Committee of Ningbo University.

\subsection{Procedure}

The tests were performed in the Sports Biomechanics Laboratory of Ningbo University. Strapping was applied to the metatarsal area in an "8-shaped" pattern (Fig.1) with elastic compressive bandage (Fig.2, width: $2.5 \mathrm{~cm}$, thickness: $1.5 \mathrm{~cm}$ ). In this study, strapping pressure was added via increasing twining laps, and it was measured with Novel Pliance (Novel, Germany). The relationship between laps and mean pressure is presented in Fig.2 Three conditions were designated as 0kpa (non-strapping, NS), 4-6kpa (moderate strapping, MS) and 10-12kpa (high strapping, HS). In addition, to ensure consistent motion quality in jump, subjects were asked to wear common shoes that they always use in competitions and training. All the participants were given at least ten minutes before each jump task to become accustomed to each strapping condition. During the test, subjects were instructed to stand on a force platform (Kistler, Germany) and to complete vertical jump from a squat posture with maximal power. To avoid energy gains associated with trunk and upper limb actions, instruction was also given requiring them to minimise the trunk action by attempting to keep it as vertical as possible and to put their hands on hips. Different strapping magnitudes were randomly selected and acceptable trials under each condition were repeated five times.

\section{Insert Fig. 1}

\section{Insert Fig. 2}

Three-dimensional kinematic data were collected using a Vicon 8-camera motion analysis system (Oxford Metrics Ltd., Oxford, UK) at frequency of $200 \mathrm{~Hz}$. Sixteen 
reflective markers (diameter $14 \mathrm{~mm}$ ) were attached on anatomical landmarks of both left and right lower limbs including: anterior-superior iliac spine, posterior-superior iliac spine, lateral mid-thigh, lateral knee, lateral mid-shank, lateral malleolus, second metatarsal head and calcaneus. A 8-channel surface EMG equipment (ME6000, Mega Electronics, Finland) was adopted to record muscle activity of tibialis anterior (TA), medial gastrocnemius (MG), rectus femoris (RF), biceps femoris (BF) at sampling rate of $1000 \mathrm{~Hz}$. Two measuring electrode slices (diameter of $10 \mathrm{~mm}$ ) were placed on positive and negative testing muscle bellies separately and the line between two slice centres was parallel to the muscle fiber direction with an inter-distance of $20 \mathrm{~mm}$. One referential electrode slice was placed on another muscle. The skin patch where the electrode slices were pasted were shaved and disinfected. All kinetic, kinematic and EMG data collection processes were conducted simultaneously.

\subsection{Data analysis}

A completed jump motion in this study was divided into three key events as take-off, flight and landing based on GRF values. The durations of each stage were obtained from force-time curves. The maximal jump height was determined according to the law of energy conservation (1) and the theorem of momentum (2). Impulse (F $\Delta \mathrm{t})$ during take-off stage could be calculated by integrating the force-time curve. All calculations were processed in MATLAB programs (MathWorks Inc., Cambridge, MA, USA). The percentage of average root mean square (RMS) by the maximal RMS among five trials was calculated to evaluate the muscle activation level. Peak joint angles in three planes of the right leg and RMS percentages (RMS \%) during take-off and landing stages were used for analysis.

$m g h=\frac{1}{2} m v_{i}^{2}$

$\mathrm{F} \Delta \mathrm{t}=\sum m_{i}^{\prime} v_{i}^{\prime}-\sum m_{i} v_{i}$

The stage between the beginning of take-off and the end of rising was selected, where 
$\mathrm{h}$ is the maximal jump height, $\mathrm{F} \Delta \mathrm{t}$ is impulse during this stage, $v_{i}$ is the velocity at beginning, and $v_{i}{ }^{\prime}$ is the velocity in the end $(0 \mathrm{~m} / \mathrm{s})$.

\subsection{Statistical analysis}

All statistical tests were conducted using SPSS 17.0 (SPSS Inc., Chicago, IL, USA). One-way repeated measures analysis of variance (ANOVA) was used to compare the duration of each divided stage, the maximal jump heights, joint angles and RMS\% across strapping conditions. A Scheffe's contrast post hoc test was applied to further determine the differences between different paired conditions. Statistical significance level was set at $5 \%$ level.

\section{Result}

\subsection{The maximal jump height}

Fig. 3 presents the maximal jump heights under three conditions of NS, MS and HS. Overall, vertical jump performance showed an improvement with MTP strapping. The average maximal height of NS group was $35.2 \mathrm{~cm}$, which was significantly less than that of $37.5 \mathrm{~cm}$ of the HS group. The data for MS group is, on average, $1.7 \mathrm{~cm}$ higher than the NS group and $0.6 \mathrm{~cm}$ less than the HS group; however, the differences between both comparisons were not significant. The height increased $4.8 \%$ and $1.6 \%$ when comparisons were made between NS and MS, MS and HS, respectively.

\section{Insert Fig.3}

\subsection{Angles of lower limb joints}

No differences were found in the duration of take-off (0-22\%), flight (23-51\%) and landing (52-100\%) across three strapping conditions. Fig.4 shows the curves of hip, knee and ankle angles in three dimensions during one jump motion. Table 1, 2, 3 lists peak angles of hip, knee and ankle during two key events of take-off and landing respectively. 


\subsubsection{Ankle}

In the sagittal plane, angles under three conditions were almost the same. In the frontal plane, compared with the control group, the MS group showed smaller inversion degree during take-off and the peak inversion presented statistical significance. In the transvers plane, curves of HS and NS were similar during take-off but different from that of MS which had a larger internal rotation degree, and the peak external rotation during take-off stage decreased significantly. Also, much smaller peak external rotation was observed under MS condition during landing.

\subsubsection{Knee}

In the sagittal plane, three curves are comparable during whole jump motion. While the peak flexion angle was significantly larger under HS in comparison with NS during take-off. In the frontal plane, although there was no significant difference between each corresponding paired conditions, the peak adduction of HS was slightly higher during take-off. In the transvers plane, the peak internal rotation and external rotation at propulsion are larger under two strapping conditions.

\subsubsection{Hip}

In the sagittal plane, hip flexion-extension was not affected by metatarsal strapping in general. The peak flexion angles under MS and HS were comparable during take-off but larger than that of NS, however, the differences failed to reach a significant level. In the frontal plane, angles for NS, MS and HS were almost the same except for slightly higher peak abduction under MS condition during take-off. In the transverse plane, the curves exhibited obvious differences between NS, MS and HS during take-off, flight and the first half of landing stage. During landing, the peak internal rotation increased as the strapping magnitude increased without significance.

\section{Insert Fig.4}




\subsection{EMG}

During take-off, significant differences of RMS\% were found in TA and BF. Compared with NS, TA RMS\% under MS condition decreased by $16 \%(\mathrm{P}=0.013)$ and BF RMS\% under HS condition decreased by $12 \%(\mathrm{P}=0.018)$. The differences of MG and RF RMS\% between strapping and non-strapping conditions were insignificant (Fig.5 a). During landing, significance only presented in TA RMS\% between NS and HS conditions (Fig.5 b). In comparison with NS, RMS\% increased by $23 \%$ ( $\mathrm{P}=0.011$ ) under HS. There were no significant differences in RMS\% values between any conditions referring to other three muscles (Fig.5 b).

Insert Fig. 5

\section{Discussion}

This study is the first systematic work to investigate the effect of metatarsal strapping on vertical jump performance, lower limb kinematics and EMG. The key results from the data of 12 male subjects shows that increasing strapping pressure from 4-6kpa to 10-12kpa could provide performance advantages for vertical jump height. The maximal jump height was increased $2.3 \mathrm{~cm}$ under $\mathrm{HS}$ in comparison with NS, which almost matched the reported increasing jump height $(2.4 \mathrm{~cm})$ when performing a maximal-effort countermovement jump wearing compression shorts (Doan et al., 2003). Two possibilities could have contributed to this improvement. Firstly, the elasticity of the bandage or the garment may increase the rigidity of the foot or hip segment, which enhances the propulsive force at push-off (Fayson et al., 2013). Secondly, compressive garments have been known to be effective in improving proprioception, which is beneficial to address jump technique (Kraemer et al., 1998; Perlau et al., 1995). Another previous theoretical study suggested that the increase in jump height was the consequence of the reduction of energy loss at MTP (Stefanyshyn \& Nigg, 1998; Stefanyshyn \& Nigg, 2000), this may also partially explains the performance improvement in the present study. Similar result that the very stiff shoe could increase jump height by $1.7 \mathrm{~cm}$ than the control shoe was 
reported in a previous research (Stefanyshyn \& Nigg, 2000). The relatively larger jump height increase in this study may indicate that increasing strapping pressure has more obvious effect on metatarsals than the approach of increasing midsole stiffness. The metatarsal strapping approach is also much simpler technically and economic in terms of facilities.

The test data showed that the influence of metatarsal strapping depends on the level of strapping pressure. As discussed above, the HS condition presented clear beneficial effects. By contrast, difference in jump height was not significant when comparison was made between NS and MS conditions, although the average height under MS was relatively higher. Regarding to data from individual participant, jump height under MS varied considerably between the subjects. This indicates that strapping magnitude of 4-6kpa is not sufficient. When the strapping pressure was increased to $10-12 \mathrm{kpa}$, the increasing height became more significant. In addition, the rate of jump height increase decreased as the strapping pressure was further increased. This suggests that that the jump performance can only be enhanced over a finite range of strapping pressure, beyond which performance cannot be further improved.

As a multi-segmental system, intervention on metatarsals may cause kinematic compensation of other lower limb joints; fortunately, no adverse results were observed generally in this study. Compensatory changes mostly occur in the frontal and transverse planes of the ankle which is located closest to metatarsals. In the sagittal plane, distinct difference only presents in the knee joint. As to the hip, the influence of metatarsal strapping in all three planes is not significant.

Compression equipment like knee neoprene sleeve, ankle tape and compressive garments have been demonstrated to improve joint position sense of these joints respectively by neuromuscular control (Kraemer et al., 1998; Birmingham et al., 2000; Spanos et al., 2008). Metatarsal strapping may also enhance joint proprioception to increase ankle joint stability; as a result, the peak inversion and external rotation 
during take-off both decreased significantly in this work. At the same time, the significantly decreased peak external rotation during landing could be considered as a reaction to the comprehensive integration of compensatory postural adjustments to stabilize this joint (Sousa et al., 2012) and help to prevent injuries. However, the data in this work showed that all significant changes in ankle exposed under MS condition rather than HS. This is probably due to the different effects of strapping magnitude on proprioception which is an essential process to motor control (Riemann \& Lephart, 2002). As shown in the data, in the sagittal plane, ankle dorsiflexion decreased slightly under strapping without significance. As to the knee, the peak flexion angle during take-off increased significantly with HS, which was similar to the effect of compression shorts that lowered the squat depth to increase impulse during the concentric phase of the jump (Doan et al., 2003). On the contrary, Moran et al. (2007) found no significant difference in jump height between two knee flexion degrees $\left(70^{\circ}\right.$ versus $90^{\circ}$ ) at outset and considered larger peak knee flexion as a helpless factor to performance improvement during a squat jump. The different durations of squat phase in these tests may lead to the adverse results observed. Apart from metatarsals, there was no significant effect of this small range strapping on hip angles, while a subtle change in the transverse plane was detected. The result in this work showed that the joint average rotation degree shifted to a more internal direction with increasing strapping magnitude. Compared with the first metatarsal, the fifth metatarsal possessed smaller anatomic cross-section (Green \& Briggs, 2013), which easily lead to medial shift of plantar pressure when five metatarsals were restricted in order to reduce weight bearing on the fifth metatarsal, as a linking reaction, the larger internal rotation of the hip could be explained. While individual variation showed to be dispersive in this study, but in each case, metatarsal strapping did increase the maximal jump height without negative kinematic changes of other lower limb joints.

Previous work showed that vertical jump height could be predicted with EMG (Verma \& Lane, 2000). Ancillao et al. (2014) revealed that there was a very high correlation between EMG and jump height. The current research tested muscle activity from TA, 
MG, BF and RF used RMS\% for evaluation of muscle latency. According to statistical analysis, RMS\% of TA and BF changed significantly when metatarsals strapping is used.

It is known that, during take-off, MG and RF play the role as agonist with concentric contraction while TA and BF play the role as antagonist with eccentric contraction (McBride et al., 2008). The data of this work showed that there were no significant differences in RMS\% of MG and RF between paired conditions during this stage, implying insignificant changes of energy generation at ankle and knee. The proprioception appeared to be more sensitive to MS magnitude and the significant difference of TA activity existed between MS and NS conditions. In compatible with kinematic changes in ankle, the smaller TA RMS\% was responsible for the reduction of ankle peak inversion to some extent. Conversely, BF RMS\% obviously decreased while the peak knee flexion increased with HS. This is probably due to the fact that knee joint position sense increased with strapping. On the basis of the reduced EMG of antagonists (TA and $\mathrm{BF}$ ), muscle coordination was possibly optimised with strapping on metatarsals, which is in agreement with the effect of compression sleeve on the knee (Kuster et al., 1999).

During landing, agonists and antagonists are exactly opposite to those during take-off. Based on the test data, only TA RMS\% was affected by strapping, a significantly increasing height with HS during this stage was observed. It is known that TA starts with its tendon on the first metatarsal; therefore, strapping on these bones may trigger the excitability of the muscle (Romkes et al., 2006) particularly at impact. Without significance, muscle activity of MG, RF and BF all exhibited a decrease with HS, this could be a beneficial change in terms of reducing tissue injury or fatigue and enhancing performance in repeated jumps. Consistent with result of the other published data, no significant differences were seen in muscle activity of MG between different strapping conditions, as well as BF and RF in this work (Roy et al., 2006). Additionally, EMG was only recorded from two flexors (TA and BF) and two 
extensors (MG and RF). It is possible that other muscles (i.e., lateral gastrocnemius, medial vastus, lateral vastus and so on) may also have been influenced by metatarsal strapping, but these data are absent. Furthermore, if strapping conditions affect the mechanical behaviour of the contractile or elastic elements of muscle tendon unit, a proper explanation would likely require a computational model of the muscle to estimate the active state and internal muscle behaviour (Jacobs et al., 1993).

In conclusion, this work shows that metatarsal strapping can be considered as an effective strategy to improve vertical jump performance. With a strapping pressure range of $10-12 \mathrm{kpa}$, the maximal jump height was increased significantly $(2.3 \mathrm{~cm})$. Changes of joint angles were found to be dependent on strapping magnitude slightly, and no obviously negative or positive compensations were observed in joints of ankle, knee and hip. The effect on EMG was found to be primarily located in TA. With strapping, TA RMS\% decreased during take-off and increased during landing. It is important to note that neither the moderate nor the high strapping condition adopted in this work is the optimal recommendation, and additional magnitudes should be tested. In addition, the underlying mechanisms that attribute to the improvement are still not fully understood. Further study should probe into energy generation and absorption at each lower limb joint including MTP under different strapping conditions. Further works on muscle activity should also involve more relative muscles. The information may be extremely useful to athletes, coaches, and sports equipment manufacturers for performance improvement.

\section{Acknowledgements}

The study sponsored by National Natural Science Foundation of China (81301600), K.C.Wong Magna Fund in Ningbo University, and Ningbo Natural Science Foundation (2013A610262). 


\section{References}

Ancillao, A., Galli, M., Rigoldi, C., \& Albertini, G. (2014). Linear correlation between fractal dimension of surface EMG signal from Rectus Femoris and height of vertical jump. Chaos, Solitons \& Fractals, 66, 120-126.

Birmingham, T. B., Inglis, J. T., Kramer, J. F., \& Vandervoort, A. A. (2000). Effect of a neoprene sleeve on knee joint kinesthesis: influence of different testing procedures. Medicine and science in sports and exercise, 32(2), 304-308.

Chatard, J. (1998). Elastic bandages, recovery and sport performance. Health and Protective Textiles, 2, 79-84.

De Glanville, K. M., \& Hamlin, M. J. (2012). Positive effect of lower body compression garments on subsequent $40-\mathrm{kM}$ cycling time trial performance. The Journal of Strength \& Conditioning Research, 26(2), 480-486.

Doan, B., Kwon, Y. H., Newton, R., Shim, J., Popper, E., Rogers, R., Kraemer, W. (2003). Evaluation of a lower-body compression garment. Journal of sports sciences, 21(8), 601-610.

Fayson, S. D., Needle, A. R., \& Kaminski, T. W. (2013). The effects of ankle Kinesio ${ }^{\circledR}$ taping on ankle stiffness and dynamic balance. Research in Sports Medicine, 21(3), 204-216.

Goldmann, J. P., \& Brüggemann, G. P. (2012). The potential of human toe flexor muscles to produce force. Journal of anatomy, 221(2), 187-194.

Green, S., \& Briggs, P. (2013). Flexion strength of the toes in the normal foot. An evaluation using magnetic resonance imaging. The foot, 23(4), 115-119.

Stefanyshyn, D. J., \& Nigg, B. M. (1998). Contribution of the lower extremity joints to mechanical energy in running vertical jumps and running long jumps. Journal of sports sciences, 16(2), 177-186.

Jacobs, R., Bobbert, M. F., \& van Ingen Schenau, G. (1993). Function of mono-and biarticular muscles in running. Medicine and science in sports and exercise, 25, 1163-1163.

Kemmler, W., von Stengel, S., Köckritz, C., Mayhew, J., Wassermann, A., \& Zapf, J. (2009). Effect of compression stockings on running performance in men runners. The Journal of Strength \& Conditioning Research, 23(1), 101-105.

Kraemer, W. J., Bush, J. A., Newton, R. U., Duncan, N. D., Volek, J. S., Denegar, C. R., Sebastianelli, W. J. (1998). Influence of a compression garment on repetitive power output production before and after different types of muscle fatigue. Research in Sports Medicine: An International Journal, 8(2), 163-184.

Kuster, M. S., Grob, K., Kuster, M., Wood, G. A., \& Gächter, A. (1999). The benefits of wearing a compression sleeve after ACL reconstruction. Medicine and science in sports and exercise, 31(3), 368-371.

Laroche, D., Pozzo, T., Ornetti, P., Tavernier, C., \& Maillefert, J. (2006). Effects of loss of metatarsophalangeal joint mobility on gait in rheumatoid arthritis patients. Rheumatology, 45(4), 435-440.

McBride, J. M., McCaulley, G. O., \& Cormie, P. (2008). Influence of preactivity and eccentric muscle activity on concentric performance during vertical jumping. The 
Journal of Strength \& Conditioning Research, 22(3), 750-757.

Moran, K. A., \& Wallace, E. S. (2007). Eccentric loading and range of knee joint motion effects on performance enhancement in vertical jumping. Human movement science, 26(6), 824-840.

Nigg, B. M., Stefanyshyn, D. J., \& Denoth, J. (2000). Work and energy mechanical considerations. In B.M.Nigg, B.R.Macintosh and J.Mester (eds.), Biomechanics and Biology of Human Movement. (pp.5-18). Champaign, IL. HumanKinetics.

Perlau, R., Frank, C., \& Fick, G. (1995). The effect of elastic bandages on human knee proprioception in the uninjured population. The American journal of sports Medicine, 23(2), 251-255.

Riemann, B. L., \& Lephart, S. M. (2002). The sensorimotor system, part II: the role of proprioception in motor control and functional joint stability. Journal of athletic training, 37(1), 80.

Romkes, J., Rudmann, C., \& Brunner, R. (2006). Changes in gait and EMG when walking with the Masai Barefoot Technique. Clinical Biomechanics, 21(1), 75-81.

Roy, J., \& Stefanyshyn, D. J. (2006). Shoe midsole longitudinal bending stiffness and running economy, joint energy, and EMG. Medicine and science in sports and exercise, 38(3), 562-569.

Sousa, A. S., Silva, A., \& Tavares, J. M. R. (2012). Biomechanical and neurophysiological mechanisms related to postural control and efficiency of movement: A review. Somatosensory and Motor Research, 29(4), 131-143.

Spanos, S., Brunswic, M., \& Billis, E. (2008). The effect of taping on the proprioception of the ankle in a non-weight bearing position, amongst injured athletes. The foot, 18(1), 25-33.

Stefanyshyn, D. J., \& Nigg, B. M. (1997). Mechanical energy contribution of the metatarsophalangeal joint to running and sprinting. Journal of biomechanics, 30(11), 1081-1085.

Stefanyshyn, D. J., \& Nigg, B. M. (2000). Influence of midsole bending stiffness on joint energy and jump height performance. Medicine and science in sports and exercise, 32(2), 471-476.

Verma, B., \& Lane, C. (2000). Vertical jump height prediction using EMG characteristics and neural networks. Cognitive Systems Research, 1(3), 135-141.

Wu, M., Ji, L., \& Jin, D. (2001). The influence of flexion of metatarsophalangeal joint on gait characteristics. Chinese Journal of Rehabilitation Medicine, 16(6), 8-12. 


\section{Figures:}

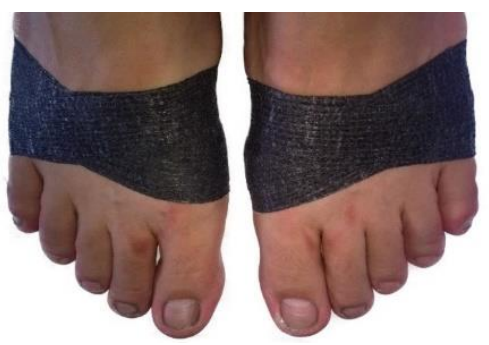

Fig.1 Strapping region and shape

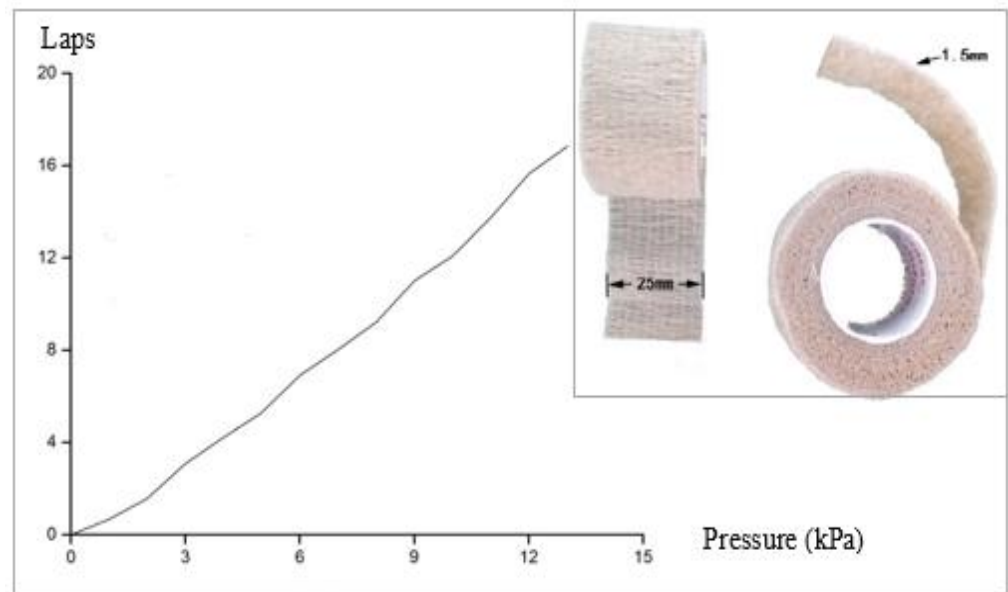

Fig.2 Elastic bandage and the relationship between laps and pressure

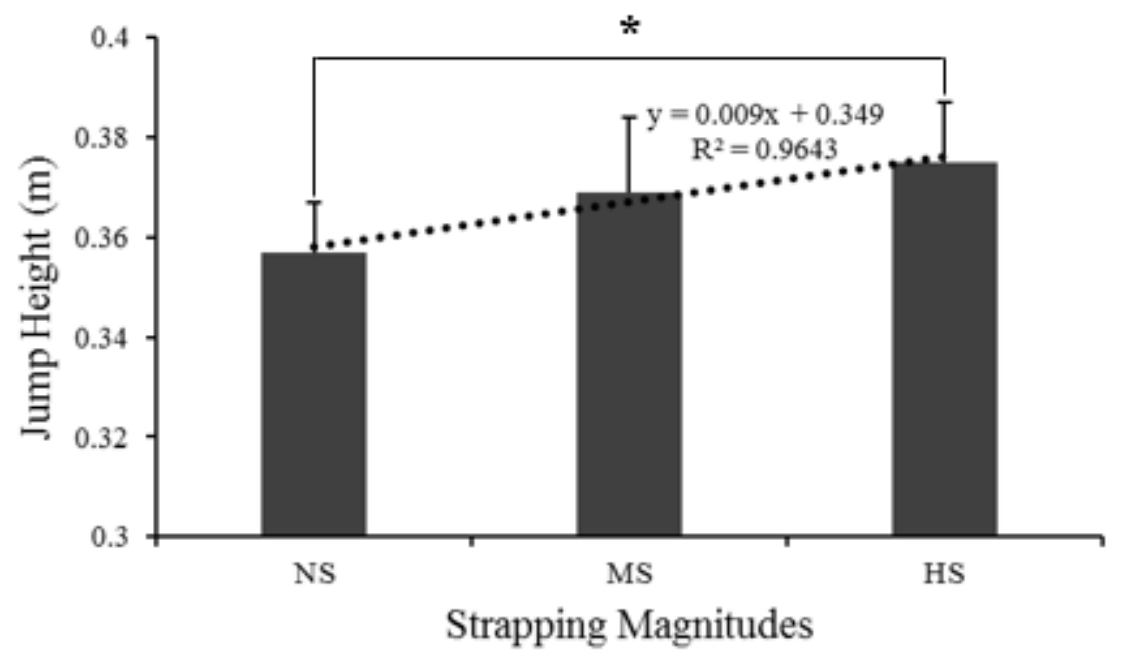

Fig. 3 Comparison of jump height between three strapping magnitudes $*$ indicates significant difference $(\mathrm{p}<0.05)$ 

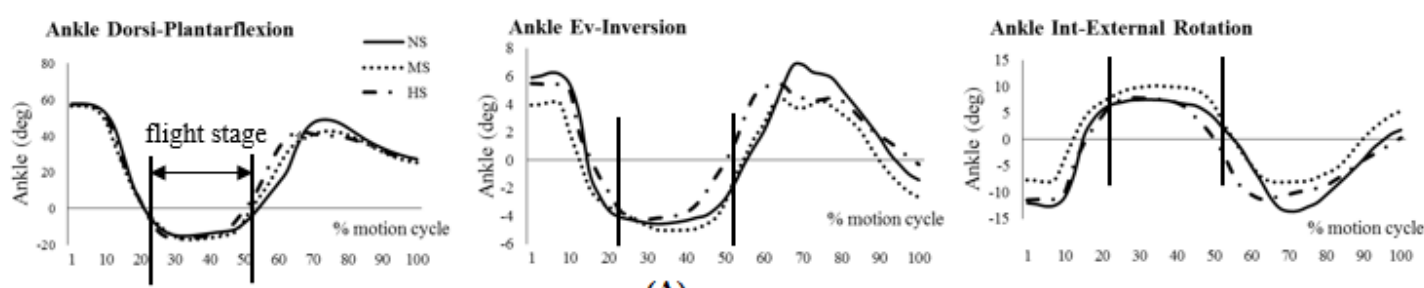

(A)
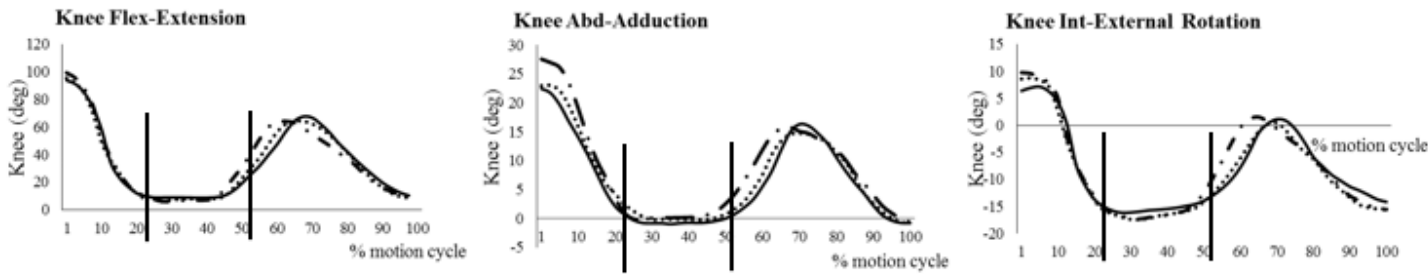

(B)
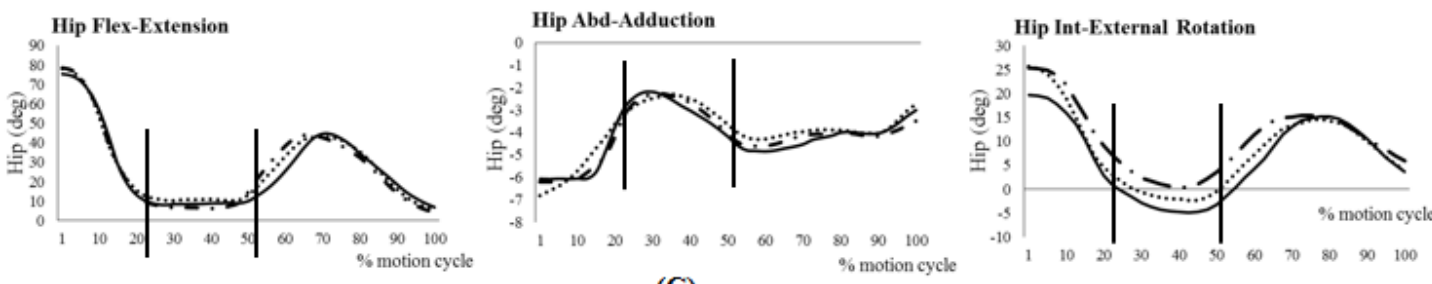

(C)

Fig.4 Joint angles of the ankle (A), knee (B), and hip (C) during one motion cycle

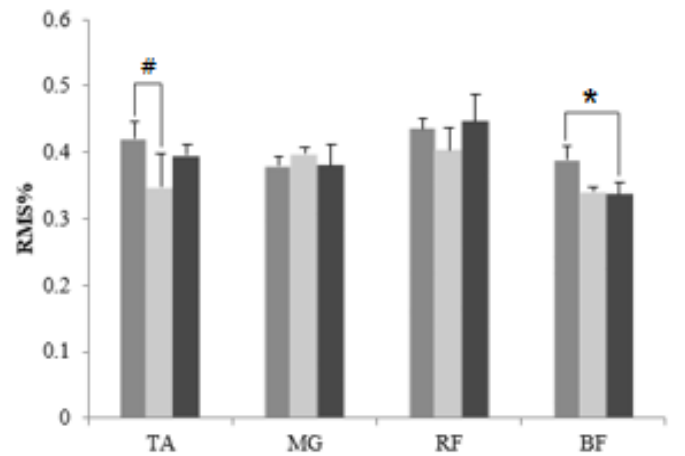

(a)

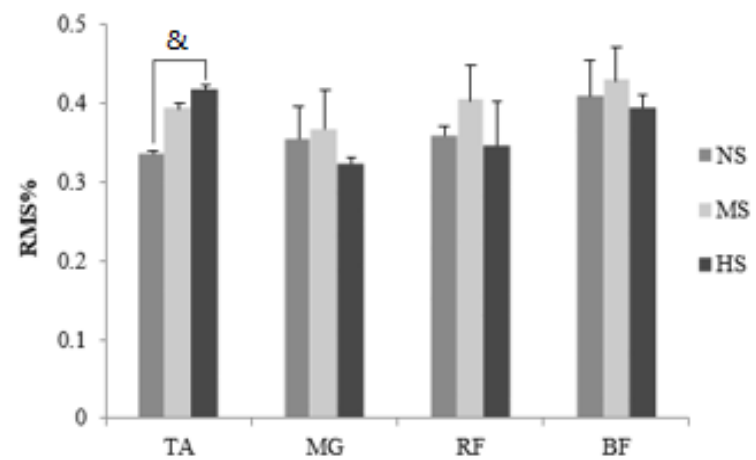

(b)

Fig.5. RMS\% of TA, MG, RF and BF under different conditions during take-off (a) and landing (b).

\#indicates significant difference between NS and MS during take-off $(P=0.013)$

* indicates significant difference between NS and HS during take-off $(P=0.018)$ $\&$ indicates significant difference between NS and HS during landing $(P=0.011)$ 


\section{Tables:}

Table 1. Peak angles of ankle in sagittal, frontal and transverse planes during take-off and landing

\begin{tabular}{|c|c|c|c|c|c|c|c|}
\hline Stages & $\begin{array}{l}\text { Strapping } \\
\text { magnitude }\end{array}$ & $\begin{array}{l}\text { Dorsi- } \\
\text { flexion }\end{array}$ & $\begin{array}{l}\text { Plantar- } \\
\text { flexion }\end{array}$ & Inversion & Eversion & $\begin{array}{c}\text { Internal- } \\
\text { rotation }\end{array}$ & $\begin{array}{c}\text { External- } \\
\text { rotation }\end{array}$ \\
\hline \multirow{6}{*}{ Take-off } & \multirow[t]{2}{*}{ NS } & 57.94 & -16.32 & 6.04 & -3.90 & 5.23 & -12.28 \\
\hline & & $(3.17)$ & (1.14) & $(1.44)$ & (1.31) & $(1.28)$ & (2.69) \\
\hline & \multirow[t]{2}{*}{ MS } & 57.07 & -18.27 & 4.64 & -4.24 & 6.17 & -9.00 \\
\hline & & $(2.74)$ & (1.24) & $(1.63)^{\#}$ & (1.63) & (2.33) & $(2.41)^{\#}$ \\
\hline & \multirow[t]{2}{*}{ HS } & 57.67 & -17.33 & 5.46 & -3.87 & 5.36 & -11.44 \\
\hline & & $(1.60)$ & (1.74) & $(1.89)$ & $(1.27)$ & $(2.90)$ & $(2.75)$ \\
\hline \multirow{6}{*}{ Landing } & \multirow[t]{2}{*}{ NS } & 52.05 & -11.89 & 5.53 & -4.02 & 7.72 & -14.00 \\
\hline & & $(2.61)$ & (1.78) & $(2.12)$ & (1.62) & $(2.45)$ & $(2.11)$ \\
\hline & \multirow[t]{2}{*}{ MS } & 46.63 & -10.02 & 3.67 & -4.62 & 10.67 & -9.22 \\
\hline & & $(5.00)$ & $(2.05)$ & (1.92) & (1.03) & (3.38) & $(3.04)^{\#}$ \\
\hline & \multirow[t]{2}{*}{ HS } & 45.33 & -9.97 & 4.34 & -3.53 & 7.51 & -11.86 \\
\hline & & $(3.34)$ & $(2.67)$ & $(2.64)$ & (1.11) & $(2.01)$ & $(2.44)$ \\
\hline
\end{tabular}

\#indicates significant difference $(\mathrm{p}<0.05)$ between NS and MS.

Table 2. Peak angles of knee in sagittal, frontal and transverse planes during take-off and landing

\begin{tabular}{cccccc}
\hline Stages & $\begin{array}{c}\text { Strapping } \\
\text { magnitude }\end{array}$ & Flexion & Adduction & $\begin{array}{c}\text { Internal- } \\
\text { rotation }\end{array}$ & $\begin{array}{c}\text { External- } \\
\text { rotation }\end{array}$ \\
\hline \multirow{3}{*}{ Take-off } & NS & $94.06(5.98)$ & $22.48(1.17)$ & $6.39(1.10)$ & $-15.19(3.59)$ \\
& MS & $94.02(6.03)$ & $22.75(1.78)$ & $7.92(2.03)$ & $-17.67(3.11)$ \\
& HS & $99.64(7.47)^{*}$ & $25.53(1.12)$ & $9.79(2.70)$ & $-17.21(1.33)$ \\
\cline { 2 - 5 } Landing & NS & $66.60(1.08)$ & $16.02(3.29)$ & $1.04(0.11)$ & $-14.54(3.29)$ \\
& MS & $62.28(2.17)$ & $13.24(3.62)$ & $0.71(0.07)$ & $-14.75(4.39)$ \\
& HS & $65.90(2.33)$ & $14.74(1.32)$ & $1.89(0.33)$ & $-15.75(1.52)$ \\
\hline
\end{tabular}

$*$ indicates significant difference $(\mathrm{p}<0.05)$ between NS and HS. 
Table 3. Peak angles of hip in sagittal, frontal and transverse planes during take-off and landing

\begin{tabular}{cccccc}
\hline Stages & $\begin{array}{c}\text { Strapping } \\
\text { magnitude }\end{array}$ & Flexion & Abduction & $\begin{array}{c}\text { Internal- } \\
\text { rotation }\end{array}$ & $\begin{array}{c}\text { External- } \\
\text { rotation }\end{array}$ \\
\hline \multirow{3}{*}{ Take-off } & NS & $75.73(2.94)$ & $-6.06(1.65)$ & $19.62(3.49)$ & $-4.56(0.70)$ \\
& MS & $78.24(1.97)$ & $-6.89(1.06)$ & $24.37(1.17)$ & $-3.21(0.46)$ \\
& HS & $77.61(3.61)$ & $-6.19(1.63)$ & $25.38(2.97)$ & $-0.42(0.37)$ \\
\cline { 2 - 5 } Landing & NS & $43.49(2.48)$ & $-4.49(1.13)$ & $15.33(2.43)$ & $-4.23(1.63)$ \\
& MS & $44.27(5.15)$ & $-4.34(0.27)$ & $14.00(3.72)$ & $-2.79(1.57)$ \\
& HS & $44.91(3.10)$ & $-4.96(1.93)$ & $14.46(1.56)$ & $-0.22(1.54)$ \\
\hline
\end{tabular}

